\title{
3-dimensional mesh generation using the Delaunay method
}

\author{
R. Hoshiko \& M. Kawahara \\ Department of Civil Engineering, Chuo University, Japan
}

\begin{abstract}
The purpose of this research is automatic generating 3-dimensional finite element mesh using the Delaunay method. In the present age, numerical analysis is used in various problems. The finite element method is one analytical approach using the finite element mesh. But, there are problems in that large scale finite element mesh generation takes a lot of time and cost. As for the large scale finite element mesh, data are enormous, and it is almost impossible for generation only by manual labor. In addition, the mesh generators prevail, but those do not always generate meshes in response to the demand of the user. It is necessary to generate mesh for the user's purpose. The Delaunay method is a technique to divide the domain into tetrahedra by using a set of nodes that are distributed arbitrarily. This technique can be applied to generate finite element meshes of complicated configuration, that is to say, practical models. In this research, generating a Suemune tunnel and mountain-shaped analysis model using the Delaunay method is presented. But, the Delaunay method cannot dispose the concave faces and interior boundaries. When generating mesh without device, inadequate elements are generated on concave faces and interior boundaries. The mesh of the concave faces and interior boundaries cannot be generated only by the Delaunay method. These problems can be solved by Virtual nodes. The Virtual nodes represent the nodes that are generated to remove inadequate elements.
\end{abstract}

Keywords: Delaunay method, Super tetrahedron, Virtual nodes.

\section{Introduction}

In recent years, the performance of the electric computer has drastically improved and the technology of numerical analysis is highly progressed. Therefore it is possible to carry out large scale numerical simulation. For example, control water 


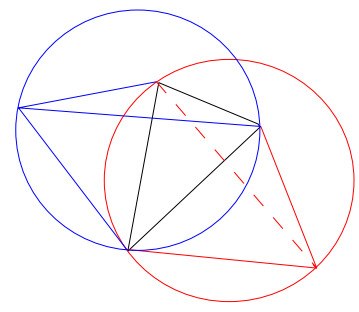

Figure 1: Delaunay tetrahedron.

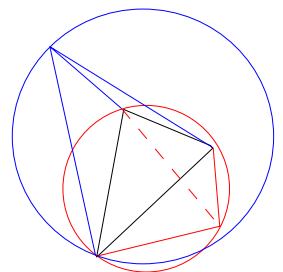

Figure 2: Not Delaunay tetrahedron.

elevation of river or bay, identification of geological boundary, etc. The finite element method has been successfully applied to solve a variety of engineering problems. On the other hand, it is dependent on the finite element mesh. The configuration of the objects to be analyzed are complicated, so that it is impossible to generate the finite element mesh by hand. Automatic mesh generation is necessary for the finite element method. The Delaunay method is an effective technique of mesh generation. They are introduced in following section.

\section{Delaunay method}

The Delaunay method is one of the technique to generate the finite element mesh. Tetrahedral elements are generated with the node group which is set arbitrarily in the computational domains. Circumscription sphere of a tetrahedron generated by the Delaunay method does not include nodes of other elements as shown in Figure 1. This is a feature of the Delaunay method. Figure 2 shows an example that is not the Delaunay tetrahedron. Using this method, a nearly regular tetrahedral mesh can be obtained. This geometry is in agreement with the preferable shape for the finite element method.

\subsection{Creation of the tetrahedron using Super tetrahedron}

A tetrahedron is known as the Super tetrahedron, if it includes a computational domain as shown in Figure 3. Super tetrahedron is useful for the computational domain into the tetrahedra. Sometimes, Super tetrahedron needs to change the size to take in the computational domain. Therefore, computational domain are covered by the tetrahedron. If a node is inserted in the computational domain, it must be in any tetrahedron. The first step of the Delaunay method is to divide the Super tetrahedron into four tetrahedra by the first node as shown in Figure 4. Basically, it is repetition that one element is divided into four tetrahedra by the inserted node.

\subsection{Division of polyhedron}

A node which is called $\mathrm{P}$ is set into the computational domain. If an element includes the node $\mathrm{P}$ is divided into four tetrahedra, the Delaunay method is not 


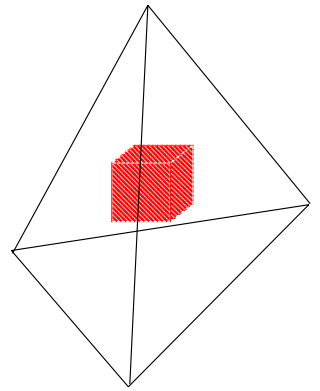

Figure 3: Super tetrahedron.

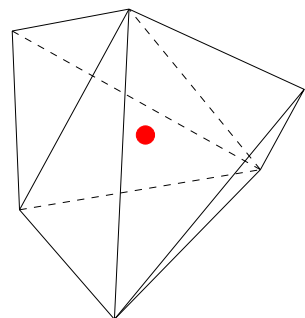

Figure 5: Polyhedron.

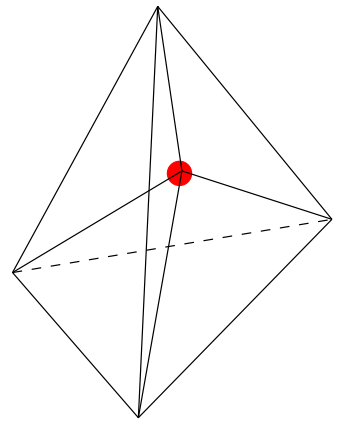

Figure 4: First step.

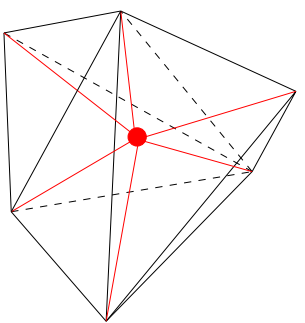

Figure 6: Generated mesh.

always satisfactory. If it is not satisfactory, these tetrahedra must be replaced according to the swapping algorithm. Elements which include the node $\mathrm{P}$ in the circumscription sphere of these elements are searched in the first step. Then, a polyhedron is generated with elements which are searched in the first step. If a polyhedron is generated, common sides of elements are removed as represented Figure 5. New elements are generated by connecting surfaces of the polyhedron and the node $\mathrm{P}$ as shown in Figure 6.

\subsection{Algorithm of the Delaunay method}

The algorithm of the Delaunay method is as follows.

1. Nodes data are input to the program.

2. Super tetrahedron is set to include a computational domain.

3. A node is inserted and search a tetrahedron which has a circumscription sphere including it.

4. Generate a polyhedron with tetrahedra which are found out.

5. Divide a polyhedron into tetrahedra.

6. If all points are inserted then Super tetrahedron is removed, else go to 3. 


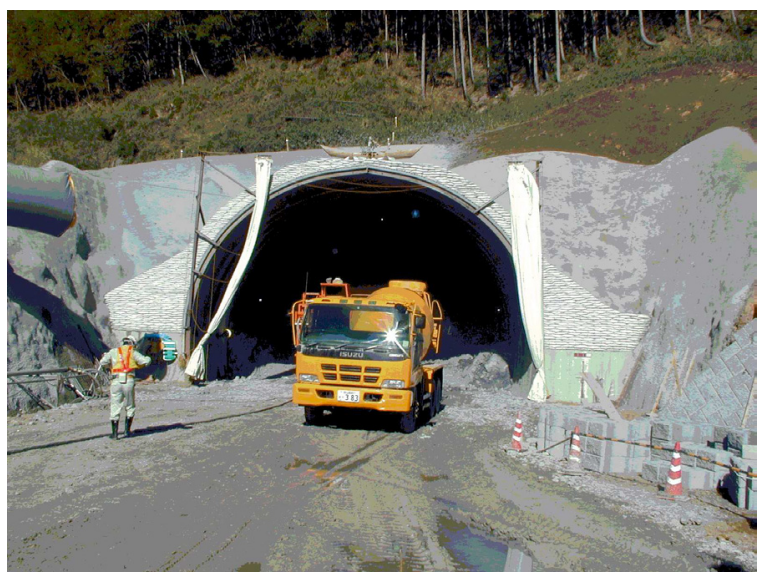

Figure 7: Suemune tunnel in Okayama, Japan.

A computer program of mesh generation is developed according to the above algorithm.

\section{Suemune tunnel and mountain model}

In this section, the Suemune tunnel model using the Virtual nodes is described. The Suemune tunnel is located in Okayama prefecture in Japan. To generate mountain and tunnel meshes, the Delaunay method is insufficient. The Delaunay method cannot dispose the complicated face. But, a lot of practical models have complicated faces. The practical model is not possible if the generation does not solve this problem. If generating practical model without taking measures, inadequate elements are generated at the tunnel mouth as shown in Figures 10 and 11. Many tricks are indispensable to generate various complicated shape. To clear up these problems, Virtual nodes are used.

\subsection{Nodal distribution of surface and Suemune tunnel}

From the provided data, nodal distribution of the tunnel is generated. The tunnels are often round in shape. To express roundness, the distance of each node becomes short. The surface of mountain and the tunnel mouth are close. In these areas, the distance between each node is incompatible. From the fine and coarse nodal distributions, the qualities of elements generated is low. Thus, it is better to subdivide the domain close to the mouth.

\subsection{Virtual nodes}

Figures 10 and 11 show inadequate elements at the tunnel mouth. These elements must be removed. Setting Virtual nodes on the tunnel mouth as shown in Figures 12 and 13, the distance is important. The Virtual nodes cannot exercise an 


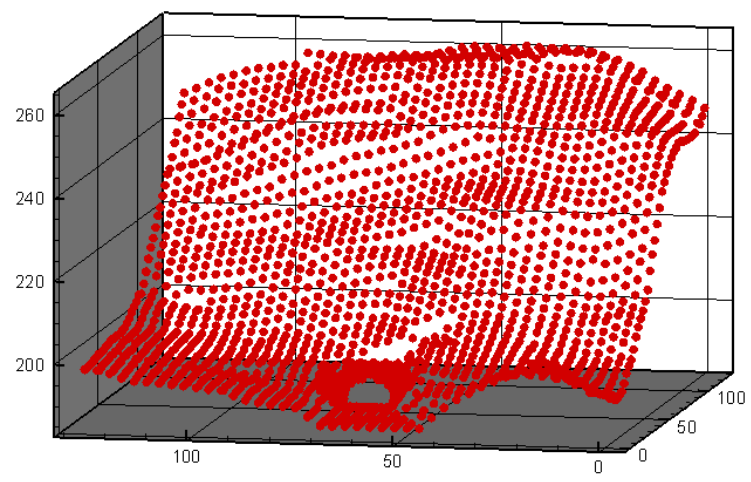

Figure 8: Nodes distributed on surface.

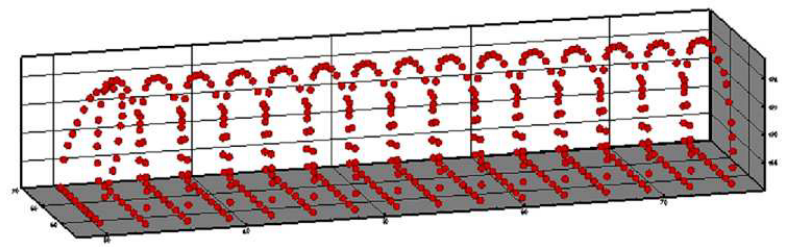

Figure 9: Nodes distributed for tunnel.

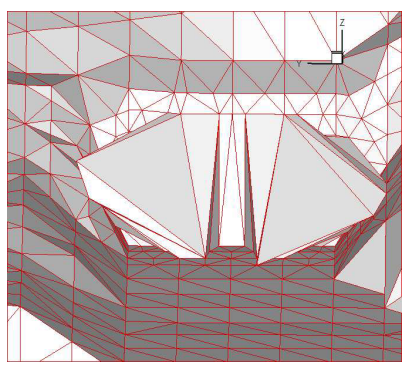

Figure 10: Inadequate elements 1.

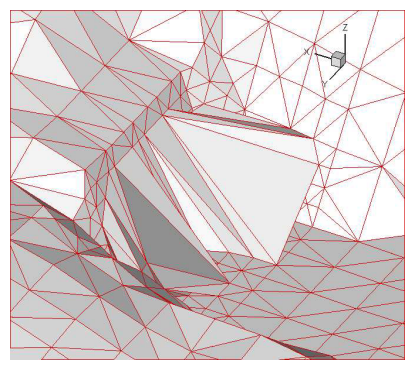

Figure 11: Inadequate elements 2.

effect if too far, or, too close. In this research, the distance between Virtual nodes is taken as an average of the interval of nodes. After the mesh generation, elements consisted of Virtual nodes are removed. Of course, Virtual nodes are removed as shown in Figures 14 and 15. The Virtual nodes are effective for not only surface but also interior boundaries. Virtual nodes and elements which consist of Virtual nodes are removed. Then, the finite element mesh which has interior boundaries is completed. 


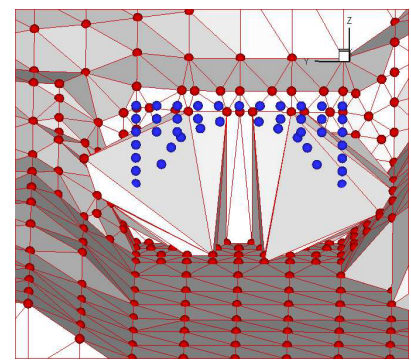

Figure 12: Virtual nodes 1.

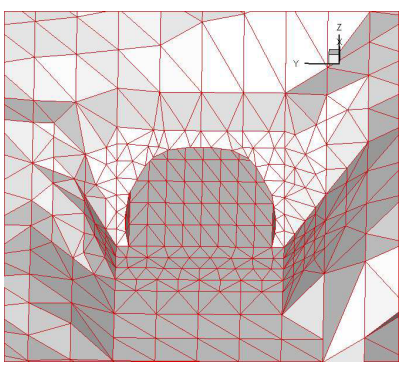

Figure 14: Tunnel mouth 1.

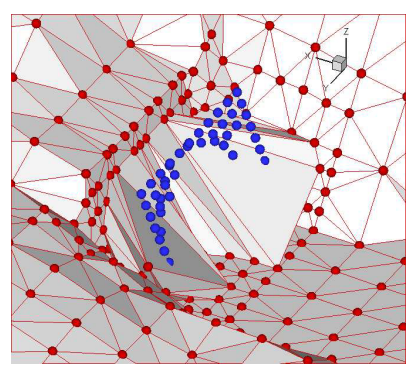

Figure 13: Virtual nodes 2.

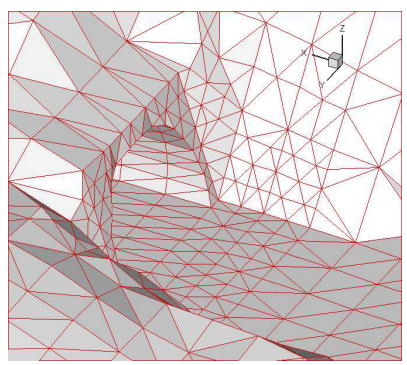

Figure 15: Tunnel mouth 2.

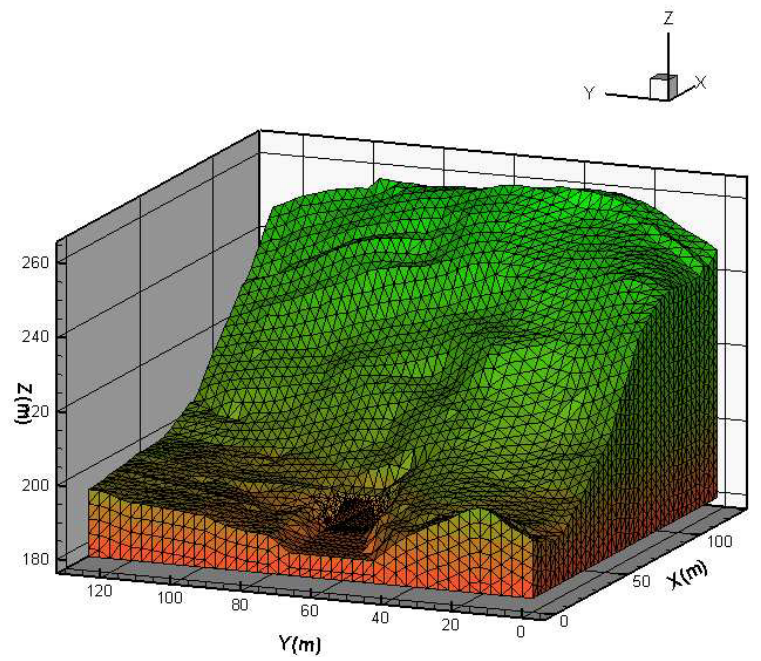

Figure 16: Completed mesh. 


\section{Conclusion}

The 3-dimensional finite element mesh can be generated using the Delaunay method. But, the Delaunay method is not perfect in generating the numerical domain. There is a problem that the Delaunay method cannot recognize the concave face and interior boundaries, yet the problem was overcome using Virtual nodes. Then, the Delaunay method is possible to divide various complicated shape domain. The Virtual nodes are effective for complicated shape domain.

\section{References}

[1] George, P. L. and Hermeline, F. Delaunay's mesh of a convex polyhedron in dimension d. Application to arbitrary polyhedra. International Journal for Numerical Methods in Engineering, 33, 975-995, 1992.

[2] Taniguchi, T. Automatic Mesh Generation for FEM, 1992.

[3] Weatherill, N. P. A method for generating irregular computational grids multiply connected planar domains. International Journal for Numerical Methods in Engineering, 8, 181-197, 1988.

[4] Thompson, J. F. A general three-dimensional elliptic grid generation system on composite block structure. Computer Methods in Applied Mechanics and Engineering 64, 377-411, 1987.

[5] Yerry, M. A. and Shephard, M. S. Automatic three-dimensional mesh generation by the modified-octree technique. International Journal for Numerical Methods in Engineering, 20, 1965-1990, 1984.

[6] Watoson, D. F. Computing n-dimensional delaunay tessellation with application to voronoi polytopes. The Computer Journal, 24(2), 167-172, 1981. 